\title{
Risk factors for respiratory hospitalizations in a population of patients with a clinical diagnosis of COPD
}

This article was published in the following Dove Press journal: International Journal of COPD

\author{
Jovica Veljanovski' \\ Daniel R Ouellette ${ }^{2}$ \\ 'Internal Medicine, Henry Ford \\ Hospital, Detroit, MI, USA; ${ }^{2}$ Division \\ of Pulmonary and Critical Care \\ Medicine, Henry Ford Hospital, \\ Detroit, MI, USA
}

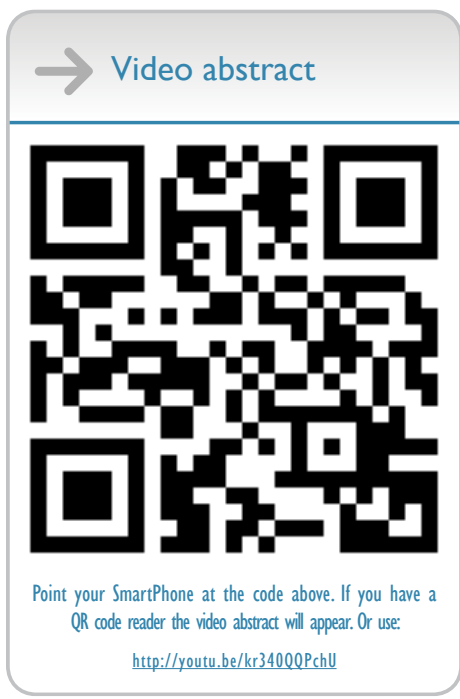

Correspondence: Daniel R Ouellette Division of Pulmonary and Critical Care Medicine, Henry Ford Hospital, K-17, 2799 West Grand Blvd., Detroit, MI 48202, USA

$\mathrm{Tel}+\mathrm{I} 3139162439$

Fax + I 3139169102

Email douellel@hfhs.org
Purpose: The purpose of this study was to examine differences between patients clinically diagnosed with COPD with and without obstruction by spirometry and to identify risk factors for respiratory hospitalizations.

Materials and methods: This is a retrospective analysis of all patients diagnosed with COPD at a large academic Internal Medicine Clinic in 2014, who had spirometry performed during the period 2013-2014. Two groups existed: one with obstruction termed classical COPD and another without obstruction. Demographics, comorbidities, prescribed medications, spirometry, respiratory hospitalization, and eosinophilia among other variables were compared between patients with and without obstruction. Risk factors for two or more respiratory hospitalizations during the period 2014-2015 were sought for both populations by both univariate and multivariate analyses. Subsequently, we studied the population without obstruction for risk factors for one or more respiratory hospitalizations first by univariate analysis and then by multivariate analysis.

Results: Among 657 patients, 210 met inclusion criteria, with 157 having obstruction on spirometry and 53 without obstruction. There was no difference between those with and without obstruction on the rate of respiratory hospitalization when using two or more respiratory hospitalizations ( $p=0.397$ ) or one or more respiratory hospitalizations $(p=0.467)$. Nontreatment risk factors associated with two or more respiratory hospitalizations by multivariate analysis included a maximum eosinophil count above the threshold of $0.5 \mathrm{~K} / \mu \mathrm{L}$ (maximum eosinophil number threshold [MENT]; $p=0.001, \mathrm{OR}=3.792,95 \% \mathrm{CI}=1.676-8.582)$ and $\%$ predicted forced expiratory volume in the first second $(p=0.031, \mathrm{OR}=0.978,95 \% \mathrm{CI}=0.959-0.998)$. In patients without obstruction, MENT above the threshold of $0.5 \mathrm{~K} / \mu \mathrm{L}(p=0.032$, OR $=5.087,95 \% \mathrm{CI}=1.147-22.557)$ was the only risk factor associated with one or more respiratory hospitalizations.

Conclusion: In a clinically diagnosed COPD population who had spirometry performed, the presence of airflow obstruction was not a risk factor for respiratory hospitalizations. The most significantly associated nontreatment factor associated with respiratory hospitalization, both in the study population as a whole and in the cohort without obstruction, was MENT above the threshold of $0.5 \mathrm{~K} / \mu \mathrm{L}$.

Keywords: chronic obstructive pulmonary disease, airflow obstruction, spirometry, eosinophilia

\section{Introduction}

COPD is a highly prevalent respiratory condition with substantial attributable morbidity and mortality. In the USA, COPD affects 24 million adults and is predicted to be the third leading cause of death by $2020 .^{1,2}$ The economic burden of COPD is substantial, with US estimated medical costs in 2020 projected to be $\sim \$ 49$ billion. ${ }^{3}$ Exacerbations of disease account for $>50 \%$ of the medical costs in the developed nations and also 
negatively impact the trajectory of this chronic disease., The prevention of exacerbations, especially hospitalizations resulting from COPD exacerbations, has become a focus for policy makers and a subject of clinical practice guidelines. ${ }^{6,7}$

COPD has traditionally been diagnosed in patients with obstructive physiology as measured by spirometry and with a compatible clinical history of smoking or other risk factors. ${ }^{8}$ Risk factors for exacerbations of COPD have been shown to include the severity of COPD and a history of exacerbations..$^{9,10}$ Recently, there has been increased awareness that some patients with a history of smoking have clinical signs and symptoms consistent with a diagnosis of COPD without having obstruction demonstrated on spirometry. ${ }^{11,12}$ Practitioners caring for patients with chronic respiratory illness may manage patients diagnosed with COPD who may not have airflow obstruction on spirometry. The goal of reducing respiratory exacerbations leading to hospitalization in a heterogeneous outpatient population clinically diagnosed with COPD is challenging because the management of patients diagnosed with COPD without spirometric evidence of obstruction is not well defined and the rate and risk factors for hospitalization for respiratory problems in such patients are not well understood.

We chose to study a population of patients from an academic Internal Medicine Clinic over a 3-year period diagnosed with COPD by their health care provider. We included patients who had spirometry performed and used Global Initiative for Chronic Obstructive Lung Disease (GOLD)'s classical threshold of forced expiratory volume in the first second $\left(\mathrm{FEV}_{1}\right)$ / forced vital capacity $(\mathrm{FVC})<70 \%$ to determine whether obstruction was present or absent. ${ }^{8} \mathrm{We}$ compared patients who had obstruction on spirometry with those who did not in terms of demographics, comorbidities, spirometry, eosinophilia, treatment, and hospitalization for respiratory problems. We hypothesized that patients with spirometric evidence of obstruction would be more likely to have hospitalization for respiratory problems compared with those who did not. We subsequently examined the study population for the association of risk factors with hospitalization for respiratory problems.

\section{Materials and methods Study design and patients}

The Henry Ford COPD K15 Study was a single-center retrospective observational study performed at an academic urban tertiary care center located in Detroit, MI, USA. The study was approved by Henry Ford Hospital Institutional Review
Board (\#10889; assessing clinical factors in patients having COPD and the impact it has on both emergency department visits and hospital admissions). A waiver for informed consent was obtained as the study posed a minimal risk to all the subjects involved, with no patient interaction and no compromise in patient rights or welfare. Patient confidentiality was maintained through password protected computer files to store data and e-mail exchange involving password protection and encryption. The study population included all patients participating in an academic Internal Medicine Clinic in 2014 with the International Classification of Diseases, Ninth Revision, diagnosis of COPD (Table S1) who had spirometry performed within the institutional database for the period 2013-2014. Subjects with active malignancy (other than stable prostate cancer) and a history of organ transplantation were excluded.

\section{Data collection and variables}

Spirometry and demographics were extracted from a pulmonary function test database for the period 2013-2014. The definition of obstructive versus nonobstructive diseases as well as the severity of COPD was taken from the GOLD ${ }^{8}$ Hospital admission data for the period 2014-2015 were extracted from the electronic medical record (EMR), after which EMR review was performed using diagnosis-related group codes to identify hospitalization for respiratory problems (Table S2). Pharmaceutical data for all outpatient medications prescribed in the period 2014-2015 were extracted using a pharmaceutical database. Medications were categorized into COPD-relevant pharmaceutical classes. EMR review for the period 2014-2015 was performed to extract patient-specific data, including the history of cardiac disease, asthma, chronic kidney disease, active malignancy, and transplantation; chronic oral glucocorticoid use; the maximum eosinophil percentage; and the maximum eosinophil count.

\section{Definitions}

Based on the presence or absence of obstruction, subjects were categorized as either having classical COPD or having nonobstructive pulmonary disease. The classical COPD cohort was defined as having obstruction on spirometry using GOLD's criteria of $\mathrm{FEV}_{1} / \mathrm{FVC}<70 \%{ }^{8}$ The nonobstructive cohort did not meet GOLD's criteria of $\mathrm{FEV}_{1} / \mathrm{FVC}<70 \%$ for obstruction, ${ }^{8}$ however received a clinical diagnosis of COPD by a board-certified internal medicine specialist, likely due to a similar clinical presentation. Comorbidities were defined as being present if listed in a physician problem list during the study period. Chronic steroid use was defined as 
prescription of oral corticosteroids other than for an acute exacerbation of COPD. Macrolide use was defined as an extended prescription of a macrolide other than for an acute exacerbation of COPD. Maximum eosinophil number (MEN) and maximum eosinophil percentage (MEP) of the total white blood count are the maximum values reported for each of these variables during the period 2014-2015 for each patient. The MEN $\geq 0.5 \mathrm{~K} / \mu \mathrm{L}$ (maximum eosinophil number threshold [MENT]) is a dichotomous variable indicating whether or not a patient's maximum value equaled or exceeded the threshold value during the period 2014-2015. The threshold was chosen by studying the relationship of the MEN to hospitalizations for respiratory problems by the examination of the patient's data. Pulmonary function test results are reported as the results $\%$ predicted compared with a standard population. ${ }^{13}$

\section{Statistical analysis}

We performed all statistical analyses by using SPSS software Version 18 (SPSS Inc., Chicago, IL, USA) with a logistical regression add-on package Version 20 (IBM, Armonk, NY, USA) and considered $p<0.05$ to be statistically significant unless otherwise specified. We used $\chi^{2}$ tests, two-sample $t$-tests, or Mann-Whitney $U$ tests, as appropriate for univariate analysis. Adjustments were not made for multiple comparisons.

Associations between risk factors and two or more hospitalizations for respiratory problems were studied in the study group first by using a univariate analysis. Nontreatment risk factors were chosen for a multivariate analysis if they were associated with respiratory hospitalizations at a significance level of $p<0.1$. MENT, MEN, and MEP demonstrated collinearity in a correlation matrix, and therefore, only the former was included in the analysis. Results were considered to be significant in the multivariate analysis if a $p<0.05$ was found. The same strategy was applied to the study of associations between risk factors and all respiratory hospitalizations in the nonobstructed cohort. Respiratory hospitalization was chosen rather than two or more hospitalizations for respiratory problems in the nonobstructed cohort because of the small number of patients with multiple respiratory hospitalizations.

\section{Results}

Of 657 patients visited the Internal Medicine Clinic during 2014 diagnosed with COPD, 242 patients (36.8\%) had spirometry performed in the period 2013-2014. A total of 210 subjects were included in the study population among whom 157 had airflow obstruction and 53 did not (selection algorithm, Figure S1). Table 1 demonstrates comparisons of study variables between those patients with and without obstruction. Table 2 displays the study population categorized by the GOLD stage ${ }^{8}$ with the rate of exacerbation leading to hospitalization indicated for each group.

We assessed the study population for risk factors associated with two or more hospitalizations for respiratory problems. A univariate analysis was performed for nontreatment (Table 3) and treatment (Table 4) risk factors with two or more respiratory hospitalizations as the outcome variable. We then performed a multivariate analysis with two or more respiratory hospitalizations as the outcome variable and considering MENT, male gender, and FEV $\%$ predicted as nontreatment risk factors. Of 210 patients, 188 were included in the analysis, with 22 cases excluded for missing MENT data. MENT above the threshold of 0.5 $\mathrm{K} / \mu \mathrm{L}(p=0.001, \mathrm{OR}=3.792,95 \% \mathrm{CI}=1.676-8.582)$ and $\%$ predicted $\mathrm{FEV}_{1}(p=0.031, \mathrm{OR}=0.978,95 \% \mathrm{CI}=0.959-0.998)$ were significantly associated with two or more respiratory hospitalizations in the model, whereas male gender $(p=0.097$, $\mathrm{OR}=1.874,95 \% \mathrm{CI}=0.892-3.937$ ) was not.

We extended our analysis to the cohort of the study population that did not have obstruction. In the nonobstructed cohort, 16 patients had one or more respiratory hospitalizations, and 7 patients had two or more respiratory hospitalizations. We used one or more respiratory hospitalizations as the outcome variable for the nonobstructed cohort and considered nontreatment (Table 5) and treatment (Table 6) risk factors in univariate analyses. We then performed a multivariate analysis with one or more respiratory hospitalizations as the outcome variable and considering MENT and $\mathrm{FEV}_{1} \%$ predicted as nontreatment risk factors. MENT above the threshold of $0.5 \mathrm{~K} / \mu \mathrm{L}(p=0.032$, $\mathrm{OR}=5.087,95 \% \mathrm{CI}=1.147-22.557)$ was significantly associated with one or more respiratory hospitalizations in the model, whereas $\%$ predicted $\mathrm{FEV}_{1}(p=0.147, \mathrm{OR}=0.971,95 \% 95 \%$ $\mathrm{CI}=0.934-1.010)$ was not.

Among 37 patients in the study population with an increased MENT, 76\% were prescribed inhaled corticosteroid (ICS) and $16 \%$ had a codiagnosis of asthma. Of 7 patients not prescribed ICS in this group, 5 patients had no respiratory hospitalizations.

\section{Discussion}

The most important findings of this retrospective study of a clinically diagnosed population of patients with COPD were that the $\mathrm{FEV}_{1} / \mathrm{FVC}$ ratio was not closely associated with respiratory hospitalization, whereas a close association 
Table I Baseline characteristics: classical COPD versus nonobstructed group

\begin{tabular}{|c|c|c|c|}
\hline Variable $(n=2 \mid 0)$ & $\begin{array}{l}\text { Classical COPD } \\
(n=157)\end{array}$ & $\begin{array}{l}\text { Nonobstructed } \\
(n=53)\end{array}$ & $\begin{array}{l}\text { Level of significance } \\
(p<0.05)\end{array}$ \\
\hline Age in years $($ mean $\pm S D)$ & $68 \pm 10$ & $64 \pm 11$ & $p=0.028 * *$ \\
\hline Active smoking & $85 / 157(54 \%)$ & $28 / 53(53 \%)$ & $p=0.869^{*}$ \\
\hline Male gender & $76 / 157$ (48\%) & $15 / 53(28 \%)$ & $p=0.01 I^{*}$ \\
\hline African American & |35//57 (86\%) & $46 / 53(87 \%)$ & $p=0.883^{*}$ \\
\hline Body surface area (mean $\pm S D)$ & $1.91 \pm 0.29$ & $2.04 \pm 0.29$ & $p=0.007^{* *}$ \\
\hline Body mass index (mean $\pm S D)$ & $28.5 \pm 8.25$ & $34.9 \pm 9.87$ & $\mathbf{p}<0.00 I^{* *}$ \\
\hline History of cardiac disease & 78/I57 (50\%) & $21 / 53(40 \%)$ & $p=0.205^{*}$ \\
\hline History of asthma & $23 / 157$ (15\%) & $5 / 53(9 \%)$ & $p=0.334^{*}$ \\
\hline History of CKD & $39 / 157$ (25\%) & $7 / 53(13 \%)$ & $p=0.077^{*}$ \\
\hline Chronic steroid use & $3 / 157(2 \%)$ & $2 / 53(4 \%)$ & $p=0.442^{*}$ \\
\hline LAMA & $109 / 157$ (69\%) & $22 / 53(42 \%)$ & $\mathbf{p}<0.00$ I* \\
\hline SAMA & $82 / 157(52 \%)$ & $24 / 53(45 \%)$ & $p=0.382^{*}$ \\
\hline SABA & |32/I57 (84\%) & $4 I / 53(77 \%)$ & $p=0.267^{*}$ \\
\hline LABA & $10 / 157(6 \%)$ & $\mathrm{I} / 53(2 \%)$ & $p=0.205^{*}$ \\
\hline SABA + SAMA & $61 / 157$ (39\%) & $23 / 53(43 \%)$ & $p=0.559 *$ \\
\hline $\mathrm{LABA}+\mathrm{ICS}$ & $112 / 157$ (7I\%) & $27 / 53(51 \%)$ & $p=0.007^{*}$ \\
\hline ICS & $34 / 157$ (22\%) & $13 / 53(25 \%)$ & $p=0.664^{*}$ \\
\hline Leukotriene receptor antagonist & $7 / 157$ (4\%) & $2 / 53(4 \%)$ & $p=0.83 I^{*}$ \\
\hline Macrolide & $69 / 157(44 \%)$ & $24 / 53(45 \%)$ & $p=0.866^{*}$ \\
\hline Phosphodiesterase inhibitor & $3 / 157(2 \%)$ & $0 / 53(0 \%)$ & $p=0.31 I^{*}$ \\
\hline Methylxanthines & $8 / 157$ (5\%) & $0 / 53(0 \%)$ & $p=0.094^{*}$ \\
\hline FVC $\%$ predicted (mean \pm SD) & $77.9 \pm 19.5$ & $72.9 \pm 19.0$ & $p=0.105^{* *}$ \\
\hline $\mathrm{FEV}, \%$ predicted (mean $\pm \mathrm{SD}$ ) & $52.3 \pm 18.4$ & $71.0 \pm 17.9$ & $\mathbf{p}<0.00$ I** \\
\hline $\mathrm{FEV}_{1} / \mathrm{FVC}($ mean $\pm \mathrm{SD})$ & $51.4 \pm 12.8$ & $76 \pm 4.9$ & $\mathbf{p}<0.00$ I** \\
\hline TLC \% predicted $(\text { mean } \pm \text { SD) })^{\mathrm{a}}$ & $105 \pm 19.3$ & $76.4 \pm 18.7$ & $\mathbf{p}<0.00$ I $^{* *}$ \\
\hline DLCO $\%$ predicted $(\text { mean } \pm \text { SD) })^{\mathrm{b}}$ & $51.7 \pm 19.6$ & $61.4 \pm 16.2$ & $p=0.003 * *$ \\
\hline MENT $\geq 0.5 \mathrm{~K} / \mu \mathrm{L}^{\mathrm{c}}$ & $72.9 \pm 19.0$ & $72.9 \pm 19.0$ & $p=0.357^{*}$ \\
\hline $\operatorname{MEP}(\%)^{c}$ & $4.66 \pm 4.46$ & $5.16 \pm 5.79$ & $p=0.550 * *$ \\
\hline $\operatorname{MEN}(K / \mu L)^{c}$ & $0.325 \pm 0.373$ & $0.4 I I \pm 0.438$ & $p=0.198 * *$ \\
\hline$\geq \mathrm{I}$ respiratory hospitalization & $56 / 157(36 \%)$ & $16 / 53(30 \%)$ & $p=0.467^{*}$ \\
\hline$\geq 2$ respiratory hospitalization & $32 / 157(20 \%)$ & $8 / 53(15 \%)$ & $p=0.397^{*}$ \\
\hline
\end{tabular}

Notes: ${ }^{*} \chi^{2}$ analysis; ${ }^{*}$ Student's $t$-test analysis; ${ }^{a} n=25$ in obstructed group and $n=17$ in nonobstructed group; ${ }^{b} n=126$ in obstructed group and $n=46$ in nonobstructed group; ${ }^{c} n=\mid 43$ in obstructed group and $n=45$ in nonobstructed group. Bold text indicates $p<0.05$.

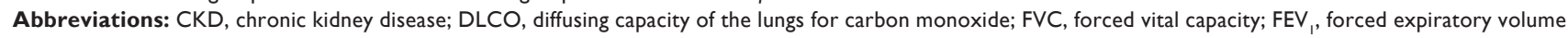
in the first second; ICS, inhaled corticosteroid; LABA, long-acting beta-agonist; LAMA, long-acting muscarinic agent; MEN, maximum eosinophil number; MENT, maximum eosinophil number threshold; MEP, maximum eosinophil percentage; SABA, short-acting beta-agonist; SAMA, short-acting muscarinic agent; TLC, total lung capacity.

was demonstrated between elevated serum eosinophils and respiratory hospitalization in all groups of patients. We demonstrated specific differences between obstructed and nonobstructed COPD patients in terms of demographics and treatment, but both groups had high rates of respiratory hospitalization. While there was a trend toward increased respiratory hospitalizations in patients with more severe COPD by GOLD stage of disease, the differences between

Table 2 GOLD $^{8}$ classification and the rate of hospital admission for respiratory illness

\begin{tabular}{|c|c|c|c|c|c|}
\hline $\begin{array}{l}\text { GOLD classification } \\
\text { of obstruction }\end{array}$ & $\begin{array}{l}\text { Number } \\
\text { of patients }\end{array}$ & $\begin{array}{l}\text { At least one } \\
\text { admission } \\
\text { (number of patients) }\end{array}$ & $\begin{array}{l}\text { At least one } \\
\text { admission, } \\
\% \text { of group* }\end{array}$ & $\begin{array}{l}\text { At least two } \\
\text { admissions } \\
\text { (number of patients) }\end{array}$ & $\begin{array}{l}\text { At least two } \\
\text { admissions, } \\
\% \text { of group* }\end{array}$ \\
\hline None & 53 & 15 & 28 & 8 & 15 \\
\hline Mild & 14 & 4 & 29 & 2 & 7 \\
\hline Moderate & 71 & 22 & 31 & 13 & 15 \\
\hline Severe & 55 & 23 & 42 & II & 18 \\
\hline Very severe & 17 & 7 & 41 & 6 & 24 \\
\hline
\end{tabular}

Note: *Differences among groups were not significant.

Abbreviation: GOLD, Global Initiative for Chronic Obstructive Lung Disease. 
Table 3 Univariate association of nontreatment risk factors with at least two respiratory hospitalizations

\begin{tabular}{|c|c|c|c|}
\hline Variable & $\begin{array}{l}\text { Patients with } \\
\geq 2 \text { hospitalizations } \\
(n=40)\end{array}$ & $\begin{array}{l}\text { Patients with } \\
\leq I \text { hospitalization } \\
(n=I 70)\end{array}$ & $\begin{array}{l}\text { Level of } \\
\text { significance } \\
(p<0.05)\end{array}$ \\
\hline Age in years $($ mean $\pm S D)$ & $66 \pm 9.6$ & $67 \pm 11$ & $p=0.492 * *$ \\
\hline Active smoking & $23 / 40(58 \%)$ & $90 / 170(53 \%)$ & $p=0.603^{*}$ \\
\hline Male gender & $23 / 40(58 \%)$ & $68 / 170(40 \%)$ & $p=0.044^{*}$ \\
\hline African American & $36 / 40(90 \%)$ & |45// 70 (85\%) & $p=0.438^{*}$ \\
\hline Body surface area (mean $\pm S D$ ) & $1.93 \pm 0.319$ & $1.95 \pm 0.291$ & $p=0.657^{* *}$ \\
\hline Body mass index (mean $\pm S D)$ & $29.6 \pm 11.1$ & $30.2 \pm 8.60$ & $p=0.7 \mid 4^{* *}$ \\
\hline History of cardiac disease & $23 / 40(58 \%)$ & $76 / 170(45 \%)$ & $p=0.145^{*}$ \\
\hline History of asthma & $3 / 40(8 \%)$ & $25 / 170(15 \%)$ & $p=0.228 *$ \\
\hline History of CKD & $9 / 40(23 \%)$ & $37 / 170(22 \%)$ & $p=0.919 *$ \\
\hline FVC $\%$ predicted $($ mean $\pm S D)$ & $73.0 \pm 2.8$ & $77.5 \pm 18.6$ & $p=0.188^{* *}$ \\
\hline $\mathrm{FEV}, \%$ predicted (mean $\pm \mathrm{SD}$ ) & $50.8 \pm 9.7$ & $58.5 \pm 19.8$ & $p=0.028 * *$ \\
\hline $\mathrm{FEV}_{1} / \mathrm{FVC}($ mean $\pm \mathrm{SD})$ & $55.3 \pm 17.0$ & $58.1 \pm 15.3$ & $p=0.290 * * *$ \\
\hline $\mathrm{FEV}_{1} / \mathrm{FVC}<70 \%$ & $32 / 40(80 \%)$ & $125 / 170(74 \%)$ & $p=0.397 *$ \\
\hline TLC \% predicted $(\text { mean } \pm \text { SD) })^{\mathrm{a}}$ & $90.3 \pm 35.1$ & $94.7 \pm 19.6$ & $p=0.614 * *$ \\
\hline DLCO $\%$ predicted $\left(\right.$ mean \pm SD) ${ }^{b}$ & $51.7 \pm 23.9$ & $54.9 \pm 17.9$ & $p=0.385^{* *}$ \\
\hline MENT $\geq 0.5 \mathrm{~K} / \mu \mathrm{L}^{\mathrm{c}}$ & $15 / 40(38 \%)$ & $22 / 148(15 \%)$ & $p=0.00 \mathrm{I} *$ \\
\hline $\operatorname{MEP}(\%)^{c}$ & $6.30 \pm 6.182$ & $4.37 \pm 4.28 \mid$ & $p=0.024^{*} *$ \\
\hline $\operatorname{MEN}(\mathrm{K} / \mu \mathrm{L})^{\mathrm{c}}$ & $0.477 \pm 0.539$ & $0.310 \pm 0.334$ & $p=0.020 * * *$ \\
\hline
\end{tabular}

Notes: * $\chi^{2}$ analysis; **Student's $t$-test analysis; $* * *$ Mann-Whitney $U$ test; ${ }^{a} n=10$ with $\geq 2$ hospitalizations and $n=32$ with $\geq 1$ hospitalization; ${ }^{b} n=33$ with $\geq 2$ hospitalizations and $n=139$ with $\geq 1$ hospitalization; ${ }^{c} n=40$ with $\geq 2$ hospitalizations and $n=\mid 48$ with $\geq 1$ hospitalization. Bold text indicates $p<0.05$.

Abbreviations: CKD, chronic kidney disease; DLCO, diffusing capacity of the lungs for carbon monoxide; FVC, forced vital capacity; FEV , forced expiratory volume in the first second; MEN, maximum eosinophil number; MENT, maximum eosinophil number threshold; MEP, maximum eosinophil percentage; TLC, total lung capacity.

groups did not achieve statistical significance. The nontreatment risk factors most closely associated with more than two respiratory hospitalizations were MEN, MEP, and MENT. MENT was the risk factor most closely associated with two or more respiratory hospitalizations when controlling for other risk factors by multivariate analysis and was the only nontreatment risk factor associated with respiratory hospitalization in the cohort of patients without obstruction.
The definition of COPD has classically included the presence of obstruction as measured by spirometry, and disease severity has been categorized based on the degree of obstruction..$^{14,15}$ This view has been challenged by the recognition that the presence of obstruction by spirometry is not a highly accurate independent risk factor for clinical outcomes and that consideration of other factors improves outcome prediction. ${ }^{8,16}$ When considering exacerbations of

Table 4 Univariate association of treatment risk factors with at least two respiratory hospitalizations

\begin{tabular}{|c|c|c|c|}
\hline Variable & $\begin{array}{l}\text { Patients } \\
\text { with } \geq \mathbf{2} \\
\text { hospitalizations }\end{array}$ & $\begin{array}{l}\text { Patients } \\
\text { with } \leq \text { I } \\
\text { hospitalization }\end{array}$ & $\begin{array}{l}\text { Level of } \\
\text { significance } \\
\text { ( } p \text {-value) }\end{array}$ \\
\hline Chronic steroid use & I/40 (3\%) & $4 / 170(2 \%)$ & $0.956 *$ \\
\hline LAMA & $32 / 40(80 \%)$ & $99 / 170$ (58\%) & $0.011 *$ \\
\hline SAMA & $37 / 40(93 \%)$ & $69 / 170(41 \%)$ & $<0.001 *$ \\
\hline SABA & $35 / 40(88 \%)$ & |38/| 70 (81\%) & $0.345^{*}$ \\
\hline LABA & $6 / 40(15 \%)$ & $5 / 170(3 \%)$ & $0.002 *$ \\
\hline SABA + SAMA & $35 / 40(88 \%)$ & $49 / 170(29 \%)$ & $<0.00 I^{*}$ \\
\hline $\mathrm{LABA}+\mathrm{ICS}$ & $34 / 40(85 \%)$ & $105 / 170(62 \%)$ & $0.005^{*}$ \\
\hline ICS & $16 / 40(40 \%)$ & $31 / 170(18 \%)$ & $0.003 *$ \\
\hline Leukotriene receptor antagonist & $4 / 40(10 \%)$ & $5 / 170(3 \%)$ & $0.047^{*}$ \\
\hline Macrolide & $28 / 40(70 \%)$ & $65 / 170(38 \%)$ & $<0.001 *$ \\
\hline Phosphodiesterase inhibitor & $2 / 40(5 \%)$ & $\mathrm{I} / \mathrm{I} 70(\mathrm{I} \%)$ & $0.034^{*}$ \\
\hline Methylxanthines & $2 / 40(5 \%)$ & $6 / 170(4 \%)$ & $0.662 *$ \\
\hline
\end{tabular}

Notes: * $\chi^{2}$ analysis. Bold text indicates $p<0.05$.

Abbreviations: ICS, inhaled corticosteroid; LABA, long-acting beta-agonist; LAMA, long-acting muscarinic agent; SABA, short-acting beta-agonist; SAMA, short-acting muscarinic agent. 
Table 5 Univariate association of nontreatment risk factors with at least one respiratory hospitalization in patients without obstruction by spirometry

\begin{tabular}{|c|c|c|c|}
\hline Variable & $\begin{array}{l}\text { Patients with } \\
\geq I \text { hospitalization } \\
(n=16)\end{array}$ & $\begin{array}{l}\text { Patients with } \\
\text { no hospitalization } \\
(n=37)\end{array}$ & $\begin{array}{l}\text { Level of } \\
\text { significance } \\
(p<0.05)\end{array}$ \\
\hline Age in years (mean $\pm S D)$ & $65 \pm 12$ & $63 \pm 11$ & $p=0.503^{* *}$ \\
\hline Active smoking & $10 / 16(63 \%)$ & 18/37 (49\%) & $p=0.354^{*}$ \\
\hline Male gender & $6 / 16(38 \%)$ & $9 / 37(24 \%)$ & $p=0.328 *$ \\
\hline African American & $15 / 16(94 \%)$ & $31 / 37$ (84\%) & $p=0.325^{*}$ \\
\hline Body surface area (mean \pm SD) & $2.0 \mathrm{I} \pm 0.338$ & $2.05 \pm 0.274$ & $p=0.639 * *$ \\
\hline Body mass index (mean $\pm S D)$ & $34.8 \pm 13.3$ & $34.9 \pm 8.17$ & $p=0.965^{* *}$ \\
\hline History of cardiac disease & $7 / 16$ (44\%) & 17/37 (46\%) & $p=0.883^{*}$ \\
\hline History of asthma & $1 / 16(6 \%)$ & $7 / 37(19 \%)$ & $p=0.237^{*}$ \\
\hline History of CKD & $1 / 16$ (6\%) & $8 / 37(22 \%)$ & $p=0.17 I^{*}$ \\
\hline FVC \% predicted (mean \pm SD) & $65.7 \pm 21.6$ & $76.0 \pm 17.2$ & $p=0.069 * *$ \\
\hline $\mathrm{FEV}, \%$ predicted (mean $\pm \mathrm{SD}$ ) & $64.3 \pm 19.3$ & $74.0 \pm 16.6$ & $p=0.068^{* *}$ \\
\hline $\mathrm{FEV}_{\mathrm{I}} / \mathrm{FVC}($ mean $\pm \mathrm{SD})$ & $76.3 \pm 5.33$ & $75.9 \pm 4.69$ & $p=0.824 * * *$ \\
\hline TLC $\%$ predicted $(\text { mean } \pm \text { SD) })^{\mathrm{a}}$ & $66.7 \pm 20.5$ & $81.6 \pm 16.3$ & $p=0.118^{* *}$ \\
\hline DLCO $\%$ predicted $(\text { mean } \pm S D)^{b}$ & $61.9 \pm 16.5$ & $61.2 \pm 16.3$ & $p=0.889 * *$ \\
\hline MENT $\geq 0.5 \mathrm{~K} / \mu \mathrm{L}^{c}$ & $7 / 16$ (44\%) & $4 / 29(14 \%)$ & $p=0.025^{*}$ \\
\hline $\operatorname{MEP}(\%)^{c}$ & $5.94 \pm 4.155$ & $4.72 \pm 6.546$ & $p=0.507^{* *}$ \\
\hline $\operatorname{MEN}(\mathrm{K} / \mu \mathrm{L})^{\mathrm{c}}$ & $0.532 \pm 0.4223$ & $0.344 \pm 0.440$ & $p=0.054 * *$ \\
\hline
\end{tabular}

Notes: ${ }^{*} \chi^{2}$ analysis; ${ }^{*}$ Student's $t$-test analysis; ${ }^{* * *}$ Mann-Whitney $U$ test; ${ }^{a} n=6$ with $\geq 1$ hospitalization and $n=1 /$ with no hospitalization; ${ }^{b} n=12$ with $\geq 1$ hospitalization and $n=34$ with no hospitalization; ${ }^{c} n=16$ with $\geq 1$ hospitalization and $n=29$ with no hospitalization. Bold text indicates $p<0.05$.

Abbreviations: CKD, chronic kidney disease; DLCO, diffusing capacity of the lungs for carbon monoxide; FVC, forced vital capacity; FEV , forced expiratory volume in the first second; MEN, maximum eosinophil number; MENT, maximum eosinophil number threshold; MEP, maximum eosinophil percentage; TLC, total lung capacity.

COPD, severity of disease as defined by the GOLD classification has been shown to be a predictor of the rate of exacerbations. ${ }^{17,18}$ However, the data suggest that patients clinically diagnosed with COPD without airflow obstruction are not simply at risk of developing COPD but may also have clinically significant disease with respiratory symptoms. ${ }^{11,12}$ In our study population, we found that there was a trend toward increased respiratory hospitalizations (Table 2) as the severity of COPD as indicated by the GOLD classification increased. This did not reach significance, probably because of the size of our study group. Obstruction by spirometry, as defined by a decreased $\mathrm{FEV}_{1} / \mathrm{FVC}$ ratio, was not a risk factor for two or more respiratory hospitalizations, although there was a weak association between a low $\mathrm{FEV}_{1}$ and two

Table 6 Univariate association of treatment risk factors with $\geq 1$ respiratory hospitalization in patients without obstructive by spirometry

\begin{tabular}{|c|c|c|c|}
\hline Variable & $\begin{array}{l}\text { Patients with } \\
\geq I \text { hospitalization } \\
(n=16)\end{array}$ & $\begin{array}{l}\text { Patients with } \\
\text { no hospitalization } \\
(\mathrm{n}=37)\end{array}$ & $\begin{array}{l}\text { Level of } \\
\text { significance } \\
(p<0.05)\end{array}$ \\
\hline Chronic steroid use & $1 / 16(6 \%)$ & $1 / 37(3 \%)$ & $p=0.534^{*}$ \\
\hline LAMA & $10 / 16(63 \%)$ & $12 / 37(32 \%)$ & $p=0.04 I^{*}$ \\
\hline SAMA & $13 / 16(81 \%)$ & $11 / 37(30 \%)$ & $\mathbf{p}<0.00$ I* \\
\hline SABA & $13 / 16(81 \%)$ & $28 / 37(76 \%)$ & $p=0.656^{*}$ \\
\hline LABA & $1 / 16(6 \%)$ & $1 / 37(3 \%)$ & $p=0.125^{*}$ \\
\hline SABA + SAMA & $14 / 16(88 \%)$ & $9 / 37(24 \%)$ & $\mathbf{p}<0.00$ I* \\
\hline $\mathrm{LABA}+\mathrm{ICS}$ & $12 / 16(75 \%)$ & $15 / 37(4 \mid \%)$ & $p=0.02 I^{*}$ \\
\hline ICS & $5 / 16(31 \%)$ & $8 / 37(22 \%)$ & $p=0.455^{*}$ \\
\hline Leukotriene receptor antagonist & $1 / 16(6 \%)$ & $1 / 37(3 \%)$ & $p=0.534^{*}$ \\
\hline Macrolide & $10 / 16(63 \%)$ & 14/37 (38\%) & $p=0.098^{*}$ \\
\hline Phosphodiesterase inhibitor & $0 / 16(0 \%)$ & $0 / 37(0 \%)$ & N/A \\
\hline Methylxanthines & $0 / 16(0 \%)$ & 0/37 (0\%) & $N / A$ \\
\hline
\end{tabular}

Notes: ${ }^{*} \chi^{2}$ analysis. Bold text indicates $p<0.05$.

Abbreviations: ICS, inhaled corticosteroid; LABA, long-acting beta-agonist; LAMA, long-acting muscarinic agent; SABA, short-acting beta-agonist; SAMA, short-acting muscarinic agent; N/A, no comparison. 
or more respiratory hospitalizations. We found a high rate of respiratory hospitalizations among patients diagnosed with COPD, but not having obstruction on spirometry. This finding is consistent with the findings of other recent works ${ }^{11,12}$ and suggests that future perspectives of smoking-related lung disease may need to incorporate such patients.

While guidelines continue to emphasize the need to obtain spirometry to confirm the diagnosis of COPD,${ }^{8}$ practicing physicians often fail to comply with this recommendation. ${ }^{19}$ Many patients with a diagnosis of COPD considered for our study did not have spirometry performed, a result that is consistent with that of Han et al, who found that only $32 \%$ of clinically diagnosed COPD patients from a large insurance database had spirometry performed. ${ }^{19}$ The implication of these data is that clinicians are diagnosing patients with COPD in the absence of recent and regular spirometry measurements and are providing treatment based on clinical signs and symptoms. Our data concerning treatment reflect that treatment strategies prescribed for patients diagnosed with COPD without obstruction on spirometry are similar to that prescribed for patients with obstruction. More data are needed in patients with smoking-related lung disease without obstruction by spirometry to understand which medications and regimens lead to improved clinical outcomes.

We considered the possibility that a different comorbidity profile between the obstructed and nonobstructed patients in our study population could account for the lack of difference in respiratory hospitalization between the two groups. Andersen et al have demonstrated in a population from Finland that patients with the COPD-asthma overlap syndrome have increased hospitalization rates when compared to patients with either COPD or asthma alone. ${ }^{20}$ Braunstein et al demonstrated that, in a population of elderly patients with chronic heart failure in the USA, the additional diagnosis of COPD was significantly associated with an increased rate of hospitalization. ${ }^{21}$ Among the patients in our study population, the prevalence of congestive heart failure, asthma, and chronic kidney was not different between the obstructed and nonobstructed groups, with trends toward a lesser prevalence of each comorbidity in the nonobstructed group. While this suggests that a difference in comorbidities does not account for the similarity in respiratory hospitalization rates between obstructed and nonobstructed patients, our assessment is limited by the fact that comorbidity data were obtained by extraction from the EMR rather than from a comprehensive clinical assessment.

Criner et al performed a systematic review of the literature and demonstrated by a meta-analysis that multiple pharmaceutical agents, including long-acting muscarinic agents (LAMA), long-acting beta-agonist (LABA), and ICS, prevent acute exacerbations of COPD. ${ }^{7}$ In our study population of patients with COPD, we found that the prescription of multiple agents was positively associated with respiratory hospitalization (Tables 4 and 6). We believe that the most likely reason for this finding is that clinicians-treated patients thought to be at risk for respiratory hospitalization more aggressively and with more agents compared with those thought not to be at risk. Our study was retrospective, and in the absence of treatment protocols or algorithms, clinician's prescribing practices were not controlled. An alternative explanation that these agents caused respiratory hospitalization in the study population seems very unlikely given the known effects of these drugs. ${ }^{7}$ Patients in the study population without obstruction by spirometry were often treated with bronchodilator and anti-inflammatory agents, though not to the same extent as those with obstruction (Table 1). The data are extremely limited regarding appropriate treatment regimens for patients with COPD without airflow obstruction, and further research is needed. ${ }^{11,12}$

Increased number and proportion of blood eosinophils have been associated with exacerbations of COPD in some but not all studies. ${ }^{22-24}$ Couillard et al found that 55 of 167 patients with COPD had eosinophilia defined as $>200$ eosinophils $/ \mu \mathrm{L}$ or $>2 \%$ of the total leukocyte count during a respiratory hospital admission for COPD and that these patients had a 3-fold risk of respiratory hospitalization over the subsequent year as well as a shorter time to the next exacerbation. ${ }^{22}$ Vedel-Krough et al studied a large cohort of COPD patients from a population database in Copenhagen and determined that an eosinophil level $>0.34 \times 10^{9}$ cells/L was associated with a 1.76-fold increase of severe exacebations. ${ }^{23}$ On the other hand, Bafadhel et al examined 243 COPD patients with respiratory hospitalization and determined that elevated eosinophil levels were not associated with an increased rate of respiratory hospitalization and that the mean length of stay for the index respiratory hospitalization was shorter than that of other patients. ${ }^{24}$ COPD patients with eosinophilia have been postulated to be more responsive to corticosteroid treatment and to have better outcomes compared with other COPD patients. ${ }^{24-26}$ We measured eosinophil levels in our study population and found that MEN, MEP, and MENT were associated with respiratory hospitalization both in the study population as a whole and in the cohort of patients without obstruction by spirometry. Our observations suggest that eosinophilia may be a risk factor for exacerbations of COPD in patients both with and without obstruction by spirometry. Most patients in our study population were prescribed either LABA/ICS or 
ICS, suggesting that the measurement of eosinophilia may be a useful marker even in patients receiving therapy.

Our study is limited by being a single-center retrospective study and the one that involves a largely urban and black population. Nearly two thirds of the available population was excluded because spirometry was not performed by clinicians. No treatment algorithms were in place to standardize the management. These elements of our study may prevent others from generalizing our results to their patients. In addition, we accepted clinicians' assignment of the diagnosis of COPD without imposing any other criteria. Although this approach may be relevant to the "real-world" management of such patients, it may have biased our patient selection toward patients with respiratory complaints and hospitalizations due to multiple causes and may be difficult to reproduce and apply in other practice settings.

\section{Conclusion}

In a population of patients diagnosed as having COPD by their clinician who had spirometry performed, the presence of airflow obstruction as measured by a reduced $\mathrm{FEV}_{1} / \mathrm{FVC}$ was not a risk factor for respiratory hospitalizations. The most significantly associated nontreatment factor associated with respiratory hospitalization, both in the study population as a whole and in the cohort without obstruction, was eosinophilia. Further research is needed to confirm these findings and to develop management strategies for COPD patients with eosinophilia and for those without airflow obstruction.

\section{Disclosure}

The authors report no conflicts of interest in this work.

\section{References}

1. Mannino DM, Homa DM, Akinbami LJ, Ford ES, Redd SC. Chronic obstructive pulmonary disease surveillance: United States, 1971-2000. MMWR Surveill Summ. 2002;51(6):1-16.

2. National Heart, Lung, and Blood Institute. Data fact sheet. Chronic obstructive pulmonary disease. 2003. Available from: http://www.apsfa. org/docs/copd_fact.pdf. Accessed January 10, 2017.

3. Ford ES, Murphy LB, Khavjou O, Giles WH, Holt JB, Croft JB. Total and state-specific medical and absenteeism costs of COPD among adults aged $\geq 18$ years in the United States for 2010 and projections through 2020. Chest. 2015;147(1):31-45.

4. Wouters EF. Economic analysis of the Confronting COPD survey: an overview of results. Respir Med. 2003;97(Suppl C):S3-S14.

5. Connors AF, Dawson NV, Thomas C, et al. Outcomes following acute exacerbation of severe chronic obstructive lung disease. The SUPPORT investigators (Study to Understand Prognoses and Preferences for Outcomes and Risks of Treatments). Am J Respir Crit Care Med. 1996;154 (4 Pt 1):959-967.

6. Centers for Medicare \& Medicaid Services. Readmissions Reduction Program. 2017. Available from: https://www.cms.gov/medicare/ medicare-fee-for-service-payment/acuteinpatientpps/readmissionsreduction-program.html. Accessed March 15, 2018.
7. Criner GJ, Bourbeau J, Diekemper RL, et al. Prevention of acute exacerbations of COPD. American College of Chest Physicians and Canadian Thoracic Society Guideline. Chest. 2015;147(4):894-942.

8. Global Strategy for the Diagnosis, Management and Prevention of COPD, Global Initiative for Chronic Obstructive Lung Disease (GOLD); 2017. Available from: http://goldcopd.org. Accessed July 26, 2017.

9. Hurst JR, Vestbo J, Anzueto A, et al. Susceptibility to exacerbation in chronic obstructive pulmonary disease. $N$ Engl J Med. 2010;363(12): $1128-1138$

10. Mullerova H, Maselli DJ, Locantore N, et al. Hospitalized exacerbations of COPD: risk factors and outcomes in the ECLIPSE cohort. Chest. 2015;147(4):999-1007.

11. Woodruff PG, Barr RG, Bleecker E, et al. Clinical significance of symptoms in smokers with preserved pulmonary function. $N$ Engl J Med. 2016;374(19):1811-1821.

12. Dransfield MT, Kunisaki KM, Strand MJ, et al. Acute exacerbations and lung function loss in smokers with and without chronic obstructive pulmonary disease. Am J Respir Crit Care Med. 2017;195(3):324-330.

13. Lung function testing: selection of reference values and interpretative strategies. American Thoracic Society. Am Rev Respir Dis. 1991;144(5): 1202-1218

14. Pauwels RA, Buist AS, Calverley PM, Jenkins CR, Hurd SS; GOLD Scientific Committee. Global strategy for the diagnosis, management, and prevention of chronic obstructive pulmonary disease: NHLBI/WHO Global Initiative for Chronic Obstructive Lung Disease (GOLD) Workshop summary. Am J Respir Crit Care Med. 2001; 163(5):1256-1276.

15. Definitions, epidemiology, pathophysiology, diagnosis, and staging. Am J Respir Crit Care Med. 1995;152(5 Pt 2):S78-S83.

16. Celli BR, Cote CG, Marin JM, et al. The body-mass index, airflow obstruction, dyspnea, and exercise capacity index in chronic obstructive pulmonary disease. $N$ Engl J Med. 2004;350(10):1005-1012.

17. Hurst JR, Vestbo J, Anzueto A, et al. Susceptibility to exacerbation in chronic obstructive pulmonary disease. N Engl J Med. 2010;363(12): $1128-1138$.

18. Mullerova H, Maselli DJ, Locantore N, et al. Hospitalized exacerbation of COPD: risk factors and outcomes in the ECLIPSE cohort. Chest. 2015;147(9):999-1007.

19. Han MK, Kim MG, Mardon R, et al. Spirometry utilization for COPD: how do we measure up? Chest. 2007;132(2):403-409.

20. Andersen H, Lampela P, Nevanlinna A, Säynäjäkangas O, Keistinen T. High hospital burden in overlap syndrome of asthma and COPD. Clin Respir J. 2013;7(4):342-346.

21. Braunstein JB, Anderson GF, Gerstenblith G, et al. Noncardiac comorbidity increases preventable hospitalizations and mortality among Medicare beneficiaries with chronic heart failure. J Am Coll Cardiol. 2003; 42(7):1226-1233.

22. Couillard S, Larivée P, Courteau J, Vanasse A. Eosinophils in chronic obstructive pulmonary disease exacerbations are associated with readmissions. Chest. 2017;151(2):366-373.

23. Vedel-Krough S, Nielsen SF, Lange P, Vestbo J, Nordestgaard BG. Blood eosinophils and exacerbations in chronic obstructive pulmonary disease: the Copenhagen general population study. Am J Respir Crit Care Med. 2016;193(9):965-974.

24. Bafadhel M, Greening NJ, Harvey-Dunstan TC, et al. Blood eosinophils and outcomes in severe hospitalized exacerbations of COPD. Chest. 2016; 150(2):320-328.

25. Suzuki M, Makita H, Konno S, et al. Asthma-like features and clinical course of chronic obstructive pulmonary disease: an analysis from the Hokkaido COPD cohort study. Am J Respir Crit Care Med. 2016; 194(11):1358-1365.

26. Pavord ID, Lettis S, Locantore N, et al. Blood eosinophils and inhaled corticosteroid/long-acting B-2 agonist efficacy in COPD. Thorax. 2016; 71(2):118-125. 


\section{Supplementary materials}

Table SI ICD-9 codes with description used to determine COPD diagnosis within Internal Medicine Clinic

\begin{tabular}{ll}
\hline ICD-9 & Description \\
\hline 491.2 & Obstructive chronic bronchitis \\
492 & Emphysema \\
496 & Chronic airway obstruction, not elsewhere classified
\end{tabular}

Abbreviation: ICD-9, International Classification of Diseases, Ninth Revision.

Table S2 ICD-9 \& DRG codes with description used to determine $\mathrm{RH}$

\begin{tabular}{|c|c|}
\hline ICD-9/DRG & Description \\
\hline 415.19 & Other pulmonary embolism and infarction \\
\hline 465.9 & Acute upper respiratory infection or unspecified site \\
\hline 466 & Acute bronchitis \\
\hline 482.1 & Pneumonia due to pseudomonas \\
\hline 482.9 & Bacterial pneumonia, unspecified \\
\hline 486 & Pneumonia, organism unspecified \\
\hline 487.1 & Influenza with pneumonia \\
\hline 487 & Influenza with other respiratory manifestations \\
\hline 491.21 & Obstructive chronic bronchitis with acute exacerbation \\
\hline 492.8 & Other emphysema \\
\hline 493.22 & Chronic obstructive asthma with acute exacerbation \\
\hline 493.9 & Asthma unspecified \\
\hline 493.92 & Asthma, unspecified with acute exacerbation \\
\hline 494. I & Bronchiectasis with acute exacerbation \\
\hline 496 & Chronic airway obstruction, not elsewhere classified \\
\hline 507 & Pneumonitis due to inhalation of food or vomitus \\
\hline 511.89 & Other specified forms of effusion, except tuberculosis \\
\hline 511.9 & Unspecified pleural effusion \\
\hline 512 & Pneumothorax \\
\hline 518.81 & Acute respiratory failure \\
\hline 518.84 & Acute and chronic respiratory failure \\
\hline 799.02 & Hypoxemia \\
\hline A3I.0 & Pulmonary mycobacterial infection \\
\hline J18.9 & Pneumonia, unspecified organism \\
\hline $\mathrm{J} 40$ & Bronchitis, not specified as acute or chronic \\
\hline J44. 1 & COPD with (acute) exacerbation \\
\hline J96.21 & Acute and chronic respiratory failure with hypoxia \\
\hline J96.22 & Acute and chronic respiratory failure with hypercapnia \\
\hline J98.01 & Acute bronchospasm \\
\hline
\end{tabular}

Abbreviations: DRG, diagnosis-related group; ICD-9, the International Classification of Diseases, Ninth Revision; $\mathrm{RH}$, respiratory hospitalization.

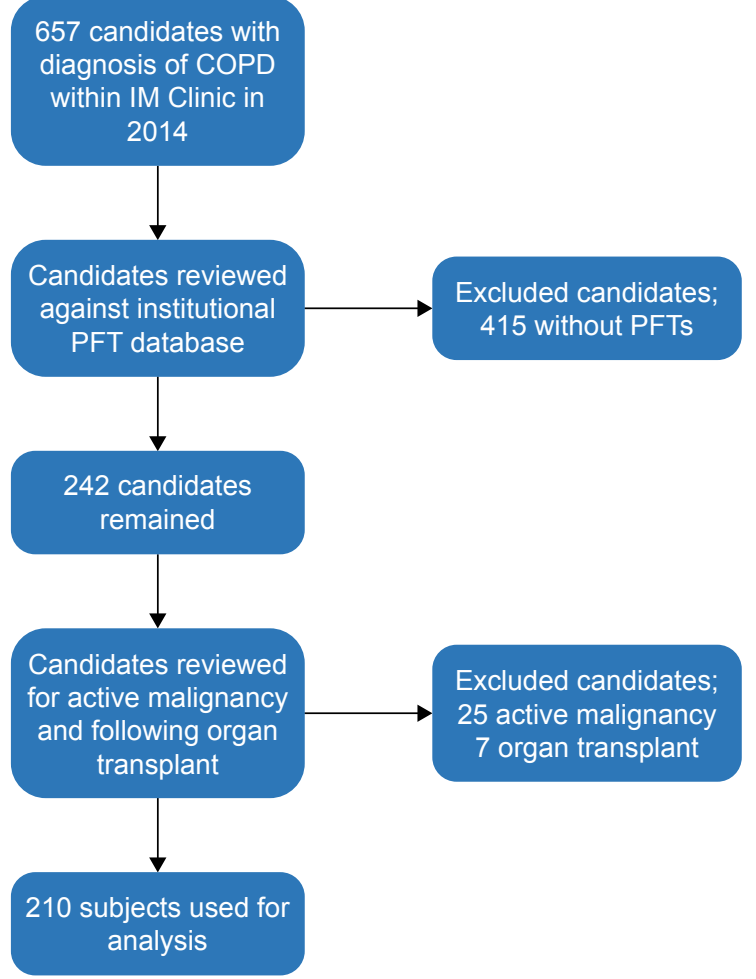

Figure SI Selection algorithm.

Abbreviations: COPD, chronic obstructive pulmonary disease; IM Clinic, Internal Medicine Clinic; PFT, pulmonary function test.
International Journal of COPD

\section{Publish your work in this journal}

The International Journal of COPD is an international, peer-reviewed journal of therapeutics and pharmacology focusing on concise rapid reporting of clinical studies and reviews in COPD. Special focus is given to the pathophysiological processes underlying the disease, intervention programs, patient focused education, and self management protocols.

\section{Dovepress}

This journal is indexed on PubMed Central, MedLine and CAS. The manuscript management system is completely online and includes a very quick and fair peer-review system, which is all easy to use. Visit $\mathrm{http}: / / \mathrm{www}$.dovepress.com/testimonials.php to read real quotes from published authors. 